\title{
La Introducción Sistemática en el Diccionario Especializado*
}

\author{
MARTA NIÑO AMO \\ Centro Internacional de Lexicografia (Universidad de Valladolid) \\ mna@emp.uva.es \\ PEDRO A. FUERTES-OLIVERA \\ Centro Internacional de Lexicografia (Universidad de Valladolid) \\ Department of Afrikaans and Dutch (University of Stellenbosh) \\ pedro@emp.uva.es
}

\section{INTRODUCCIÓN}

Aunque existen diferentes tradiciones lexicográficas podemos afirmar que para los hispanohablantes el diccionario es básicamente una herramienta de información y referencia que se construye para ser consultada de forma puntual (es decir, no se lee desde el principio hasta el final, sino que se acude a la palabra o parte del diccionario que motiva la consulta) con el objetivo de proporcionar conocimientos necesarios a sus usuarios potenciales de la forma más rápida, cómoda y útil posible. También en la tradición hispanohablante suele diferenciarse entre un diccionario, que proporciona conocimiento de lengua y una enciclopedia, que ofrece conocimientos de cosas y hechos. A pesar de que esta distinción goza de gran tradición, la investigación metalexicográfica más reciente ofrece un buen número de argumentos en contra de dicha diferenciación (Fuertes-Olivera y Tarp 2014). Por ejemplo, en el Diccionario español de contabilidad (Fuertes-Olivera y otros 2013), la entrada coste amortizado (Figura 1), registra datos propios de un diccionario tradicional (a saber, las flexiones que indican que es un nombre masculino, una definición para legos interesados o semiexpertos, y contextos de uso en los que el concepto aparece junto a otras palabras con las que forma combinaciones frecuente. Además, el usuario potencial puede acceder a la Norma Internacional de Contabilidad 39 (hipervínculo NIC 39) y a la etiqueta «E + IAS/IFRS», que indica que es un término sacado de la traducción realizada en España de las Normas Internacionales de Contabilidad. Tanto el hipervínculo como la marca lexicográfica ponen de manifiesto aspectos enciclopédicos que nos permiten argumentar, entre otras cosas, que la diferenciación tradicional entre diccionario y enciclopedia puede no ser necesaria en la época actual (Fuertes-Olivera y Niño Amo 2011).

En este artículo vamos a profundizar en la idea anterior presentando la filosofía con la que hemos construido una introducción sistemática para su incorporación al Diccionario español de contabilidad (sección 5). Para llevarla a cabo es necesario explicar las

${ }^{*}$ Este trabajo se ha llevado a cabo en el marco del proyecto financiado La Teoría Funcional de la Lexicografia: Diseño y Construcción de Diccionarios de Internet (Ref. FFI2014-52462-P). Nuestro agradecimiento al Ministerio de Economía y Competitividad por la financiación otorgada. 
características ontológicas del dominio de especialidad desde la perspectiva del usuario potencial del diccionario (sección 2), la categorización del dominio como lenguaje de especialidad (sección 3), y su viabilidad en un diccionario especializado tal y como el mismo se concibe en el marco de la teoría funcional de la lexicografía (Fuertes-Olivera y Tarp 2014; sección 4).

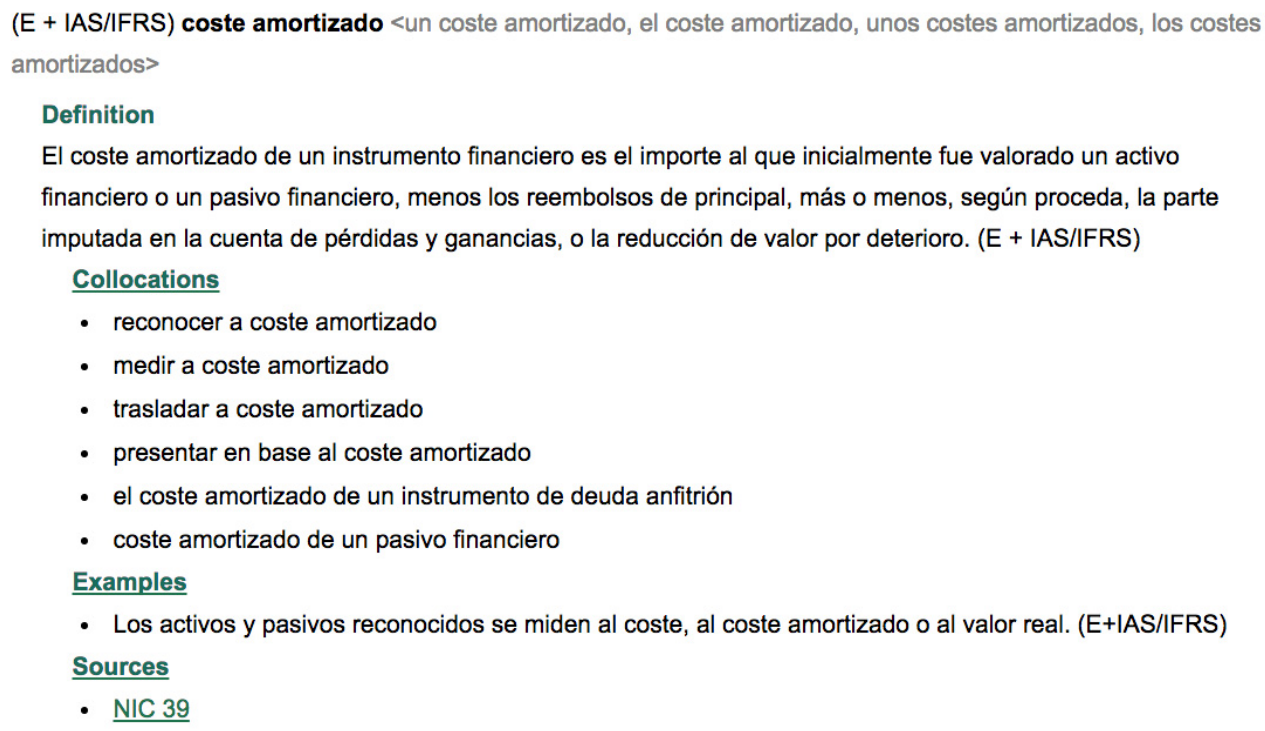

Figura 1: Coste amortizado en el Diccionario español de contabilidad

\section{LA CONTABILIDAD}

La contabilidad en el mundo actual tiene un gran protagonismo debido a la generalización de su uso como lenguaje de los negocios y a la creciente repercusión social de la información financiera. A través de la contabilidad se obtiene, o pretende obtenerse, una imagen de la realidad económica de las empresas. Pero no es solo desde un punto de vista mercantilista desde el que la contabilidad está cobrando un gran protagonismo sino también porque está relacionada con temas como el crecimiento sostenible, la responsabilidad social corporativa y otros aspectos éticos de la actividad económica, que adquieren relevancia con el cambio de siglo.

El lenguaje contable sirve como vía de comunicación de ideas y conocimientos, convirtiendo la contabilidad en una práctica social que en situaciones de crisis como la actual le otorga una visibilidad financiera que debe ser atendida y entendida tanto por los profesionales y los académicos, es decir, por expertos en la materia, como por el público en general, con menos o nulos conocimientos. La información publicada contiene términos pertenecientes a un lenguaje especializado - el lenguaje contable - que expresarán conceptos que son importantes para todas las partes, aunque pueden no estar al alcance de todos los interesados por carecer de los conocimientos precisos. 
La idea expresada en el párrafo anterior no es nueva. Diversas investigaciones han demostrado que el diccionario era (y es) un elemento esencial para trasmitir el estado actual del conocimiento en una o varias materias (Besomi 2011). Esto es posible porque el diccionario también puede enseñar al lego y al semiexperto; por ejemplo, al jurista o al periodista de prensa económica que sin ser expertos deberán comprender determinados conceptos en relación con la contabilidad para mejor cumplir sus labores profesionales. Esta es una de las funciones principales de la introducción sistemática, tal y como explicaremos en la sección 5 .

\section{LA CONTABILIDAD, LENGUAJE ESPECIALIZADO}

Dado que los conceptos contables y económicos han entrado en la corriente principal de la sociedad en general y se utilizan a menudo para enmarcar debates sobre temas polémicos, la claridad y la utilidad de la contabilidad como lenguaje especializado es cada vez más importante, ya que no se limita a la difusión y uso de información contable en términos cuantitativos, también trasmite y difunde información en términos cualitativos (Killian 2010).

El lenguaje contable como lenguaje especializado es un sistema complejo, con reglas y estructuras que se han ido ampliando y puliendo a tenor de la evolución y complejidad de la realidad económica que pretende describir. Este lenguaje que corresponde a la investigación, y a la trasmisión de esa investigación, precisa en su utilización de una formación especial en la conceptualización, clasificación y comunicación dentro de este campo de conocimiento específico.

Al igual que en otros campos del conocimiento, la contabilidad — lo que representa, tanto en su investigación como en su aplicación - es y ha sido a lo largo de su historia, dependiente de un vocabulario especializado que constituye su terminología, tanto para lograr sus objetivos como para diferenciarlo de otras disciplinas.

El lenguaje contable es una variedad de un lenguaje natural. Tales variedades se dan cuando la comunicación debe atender a propósitos específicos, con una terminología especializada (como por ejemplo, activo intangible, imagen fiel, valor razonable, etc.). En términos lingüísticos estos lenguajes suelen denominarse de formas diversas: lenguaje de género, lenguaje específico, lenguajes de especialidad, lenguajes para fines específicos e, incluso, «jerga» (Allan 2006).

Tales registros o jerga responden a unos propósitos y tienen unas implicaciones. Así, facilitan la comunicación entre los miembros de un grupo, de una profesión, lo que permite una economía de medios de expresión, por lo que estos lenguajes son eficientes y precisos. Esto lo logran «al normalizar el uso de las palabras (léxicos) y la denominación de los conceptos, [...] para que sean óptimos para la comunicación científica o profesional» (Engberg 2006: 681).

El lenguaje ordinario y el lenguaje contable no son independientes; al contrario, están estrechamente relacionados; el segundo se desarrolla a partir del primero, surge como evolución natural al adaptarse al ámbito económico. Por tanto el léxico que designa de forma concreta un concepto se convierte en término específico cuando se utiliza en el ámbito contable con sentido y significado también contable. 
La utilización de un lenguaje contable diferenciado del lenguaje ordinario permite a sus usuarios identificarse como grupo en el que pueden expresar una conciencia profesional, una unión social. El uso de ese lenguaje especial puede señalar a un individuo como miembro de esa élite intelectual o técnica, a la que puede unirse al convertirse en especialista en el ámbito respectivo, a través de la educación, la formación o la experiencia. Estas expresiones propias y específicas del lenguaje contable sirven también para amplificar la propia imagen y hacer que parezca importante lo que es, en esencia, trivial. Además la jerga especializada tiene también la pretensión de desconcertar e intimidar a la «clientela», es decir, los tecnicismos sirven para excluir a los no miembros mediante la creación de barreras (Allan 2006: 110).

La pertenencia al grupo le otorga al profesional el derecho a definir la terminología, adaptándose a los cambios que se puedan dar en la misma, consecuencia de la evolución histórica, o de distintos escenarios sociales y culturales en un mismo momento; además puede resolver sobre los conceptos y expresiones que prevalecerán, en caso de conflictos (Engberg 2006: 679-680).

Por tanto, para el registro de la contabilidad se utiliza la terminología especializada, aunque también se comparten palabras y expresiones con los no contables. Debemos ser conscientes, en primer lugar, de que los significados pueden ser distintos en el uso cotidiano en contraposición al registro especial contable (lo que origina uno de los motivos de conflicto en la comunicación) y, en segundo lugar, la terminología aún perfectamente definida, puede ser interpretada de manera diferente por diferentes grupos de personas: profesionales, legos, legisladores e investigadores, por ejemplo, además de las dificultades de tratar con términos de diferentes épocas e idiomas (lo que motiva conflictos de comunicación culturales).

La importancia del lenguaje para la teoría de la contabilidad y la metodología ha sido investigada y discutida a través de diferentes enfoques. El análisis lingüístico del lenguaje contable supone que, por ejemplo, algunas dificultades y malentendidos en la contabilidad se originan en la relación entre lenguaje contable y la lingüística. La relevancia de la lingüística en la contabilidad se puede constatar a través de la mejora de la teoría y la práctica contable al considerar las implicaciones lingüísticas en la elaboración y difusión de las primeras.

El estudio del significado lingüístico, semántico y pragmático de términos y expresiones eminentemente contables hace posible superar los problemas que la convivencia de diferentes idiomas y entornos culturales muy diversos podrían suscitar. Los malentendidos y dificultades, que se dibujan en la transmisión/recepción de información contable, debidas, entre otras, a traducciones inexactas, ambigüedades conceptuales, desconocimiento de la materia, etc., provocan problemas evitables con una clara y correcta definición de términos, expresiones y aún acrónimos, cuyas siglas pueden tener significados diferentes, en momentos o ámbitos distintos.

Las investigaciones revelan que la identificación de contabilidad, (así como cualquier otro conocimiento humano), está totalmente relacionada con el lenguaje. Las relaciones, semejanzas y diferencias de significado entre el registro especializado y el lenguaje ordinario, es decir, entre la contabilidad, la lingüística, la semántica y la 
pragmática han sido bien estudiadas y establecidas, aún desde enfoques distintos, entre otros, por Avery (1953); Haried (1972, 1973, 2002); Oliver (1974); Belkaoui (1978, 1980); Cuevas (2009; 2010), López Pérez (1999); López Pérez y Rodríguez Ariza (2002 y 2003).

\subsection{Elaboración y articulación del lenguaje contable}

El lenguaje contable reúne una serie de características terminológicas y lingüísticas concretas que ponen limitaciones narrativas y expresivas en la transmisión de conocimiento. Cualquier realidad económica sobre la que queramos informar debe acomodarse a esta estructura lingüística peculiar. Así estructurada la visión de la realidad de la que se ocupa y su configuración incide en la forma de entender la actividad económica; proporciona una guía de racionalidad al usuario e influye en la comprensión de la realidad. La racionalidad que se aplica, la gramática que se emplea y la simbología propias inciden en la visión que se puede obtener de la realidad. Para entender la información contable es preciso aprender este lenguaje específico, que es de difícil comprensión para los que no lo han estudiado (Jones y Shoemaker 1994, citados en López Pérez y Rodríguez Ariza 2003: 676).

El lenguaje contable constituye un discurso formal, sujeto a una serie de reglas y premisas en su elaboración. Las hipótesis sobre las que tradicionalmente han descansado las construcciones contables serían las reglas fundamentales que se consideran en la elaboración de la información financiera y que implícitamente entienden los usuarios expertos cuando leen elaboraciones contables (AECA 1995: 65).

En las elaboraciones contables influyen elementos de diversa naturaleza, tales como la percepción y comprensión de la realidad económica, de las capacidades individuales del que procesa la información; las habilidades, conocimientos o motivaciones del emisor; el medioambiente o contexto en el que tiene lugar la comunicación y, por supuesto, la estructura lógica inherente en este lenguaje. Cada uno de estos elementos requieren diferentes métodos de conocimiento porque pertenecen a diferentes dimensiones, aunque en conjunto forman un único proceso que se puede definir como la «articulación del lenguaje de la contabilidad» (López Pérez y Rodríguez Ariza 2002: 461).

El lenguaje contable, en ningún caso, es algo rígido; depende del uso que se haga del mismo. En este contexto, hay que tener en cuenta que los términos, que se derivan del uso, influyen a su vez en ese uso, ya que tienen un carácter convencional y van unidos al contexto social en el que se emplean. El lenguaje se acomoda a las necesidades informativas que deban ser atendidas y su configuración depende de la tradición contable en la que nos movamos, del entorno político, económico, histórico, cultural, ético o de los intereses de los diferentes grupos de usuarios, que van a configurar sistemas contable diferentes, con visiones e interpretaciones de la realidad distintas (Evans 2004: 240-243).

La hermenéutica, o interpretación, en la contabilidad se estudia para explorar la presencia de la lengua en la contabilidad. La hermenéutica ha encontrado diferentes lecturas a través del tiempo de hechos y actividades contables, explicadas de formas diferentes y con diferentes puntos de vista (Irausquín 2012). Las implicaciones de lecturas 
divergentes ocasionan distorsiones del mensaje contable. Aún teniendo en cuenta la importancia de una correcta emisión/recepción de la información contable, que puede derivar en situaciones graves de manipulación, no ya de datos, sino de información cualitativa; menos regulada pero importante en este objetivo primigenio de que la información contable sirva para la toma de decisiones racionales; son muy pocos los estudiosos contables los que hasta el momento han considerado el aspecto interpretativo del conocimiento. Las pocas explicaciones hermenéuticas de contabilidad, que se han estudiado declaran, sin embargo, que la contabilidad es un texto que requiere interpretación más allá de los verificables y comprobables datos cuantitativos.

Estos elementos, además de la intención y características personales del emisor, formadas por sus conocimientos, destrezas y motivaciones constituyen la dimensión pragmática, de uso habitual, del lenguaje contable. Estos términos deben ser conocidos y contemplados ya que afectan a la perspectiva de observación de la realidad económica, modificable en función del entorno o intereses concretos de grupos determinados; pero que no deben alterar ni las reglas básicas ni los elementos estructurales.

El lenguaje contable proporciona una terminología específica y sigue una lógica específica en cuanto a cómo el individuo desarrolla su pensamiento. Para la transmisión de los hechos económicos de los que se informa, es necesario entender el idioma y traducirlo a través del sentido de esos eventos. «El lenguaje contable es un lenguaje específico en el campo económico que tiene como objetivo representar los elementos que intervienen en el negocio y el fenómeno de la circulación del valor que se da entre ellos» (Belkaoui 1978: 97).

En realidad el lenguaje contable, para este autor, es una manifestación más de la visión débil de la hipótesis de Sapir y Whorf (Whorf 1956), que establece que la lengua a la que un hablante está expuesto influye de forma notable en la conceptualización de algo, por ejemplo, de la realidad económica de una empresa o institución. No cabe duda de que el trabajo pionero de Belkaoui es importante en tanto en cuanto es el primer autor que demuestra que las características léxicas y las reglas gramaticales que se manifiestan en los documentos contables que analiza tienen un enorme impacto sobre el comportamiento del usuario de la información contable. En concreto, Belkaoui formula cuatro proposiciones o supuestos que señalan la importancia del relativismo lingüístico de la hipótesis de Sapir y Whorf, ya que los mismos hacen hincapié en presentar el lenguaje como mediador y formador del entorno:

- Los usuarios que son capaces de realizar distinciones léxicas en el discurso contable están en disposición de analizar y/o solucionar problemas que no pueden ni analizar ni solucionar los usuarios que no han llevado a cabo dichas distinciones léxicas.

- Los usuarios que son capaces de realizar distinciones léxicas en el discurso contable están capacitados para llevar a cabo tareas (no lingüísticas) de forma más rápida y completa que los usuarios que no han llevado a cabo tales distinciones léxicas. 
- Los usuarios que son conscientes de las reglas (gramaticales) contables están más predispuestos a aceptar y valorar estilos de gestión y comportamiento diferente que aquellos usuarios que no son conscientes de dichas reglas contables.

- Las técnicas contables pueden o bien facilitar o bien dificultar algunos comportamientos de gestión.

Las operaciones económicas, pues, se traducen en un sistema lingüístico específico que sigue una cierta lógica. La articulación contable precisa, por tanto, un análisis lógico que nos mostrará el tipo de razonamiento que se sigue, y el lenguaje a utilizar, estudiando su significado, posibilitando que el resultado final (información contable) aporte la consistencia, organización y cohesión necesarias para el cumplimiento de sus objetivos de utilidad a sus usuarios.

\subsection{Evolución y cambios del lenguaje contable}

Muchos de los cambios, en el contexto del lenguaje y la estructura lógica tienen su origen en los cambios que se producen en el conjunto de la sociedad y no solo en el entorno económico. Estos cambios, justificados por necesidades sociales, pueden llegar a afectar al contenido formal y estructural de la información que se transmite.

Parker (1994) examina el origen de la terminología contable y en particular las interacciones con las palabras y los conceptos jurídicos y económicos, materias que guardan una estrecha relación con la disciplina contable. Presta especial atención a la evolución de términos y conceptos y los cambios de significado que alguno de ellos ha tenido a través del tiempo y sugiere tres razones para la introducción o la acuñación de nuevos vocablos y/o modificación de los existentes:

- Necesidad de nuevos términos. Ante situaciones de nuevos aspectos técnicos, evolución de los mismos y cambios en el conocimiento; se impone la creación de nuevos vocablos para adaptarlos a los nuevos hechos, situaciones o conceptos que deben describir.

- Términos relacionados con cambios culturales y/o de idioma. Estas situaciones suponen cambios motivados por las influencias del entorno social y cultural que una economía globalizada hace inevitable.

- Terminología para el discurso con los no contables. Con el fin de propiciar una comunicación más eficiente, se puede recurrir a la utilización de expresiones que describan de forma accesible para los no contables, hechos u operaciones que si presentan una gran dificultad. La búsqueda de un término para mas coloquial y accesible que el técnico, es el objetivo de que se pretende con la elaboración de la introducción sistemática.

La necesidad de verbalizar nuevos conceptos nace porque en una situación de cambio de las condiciones del entorno surgen nuevas reglas, principios y conceptos jurídicos y económicos $\mathrm{y}$, por ende, contables, por lo que no se tendrá un término o equivalente para describir esa nueva situación (porque si existiera el término, el concepto también hubiera tenido que existir). Por ejemplo, la última crisis financiera ha implicado la práctica imposibilidad de acceso a las tradicionales vías de financiación para las empresas y ello ha supuesto que haya sido preciso diseñar formas de financiación al 
margen del sistema bancario. Esto lleva aparejado la necesidad de nuevos términos íntimamente asociados a esas nuevas operaciones de tipo financiero: así nos encontramos con expresiones como: capital semilla, financiación ángel, peaje (en) sombra, por citar algunos que han tenido que ser creados para reflejar situaciones totalmente novedosas. La figura 2, por ejemplo, muestra la definición de peaje en sombra en el Diccionario español de contabilidad, y la utilidad de la misma para entender estas nuevas realidades:

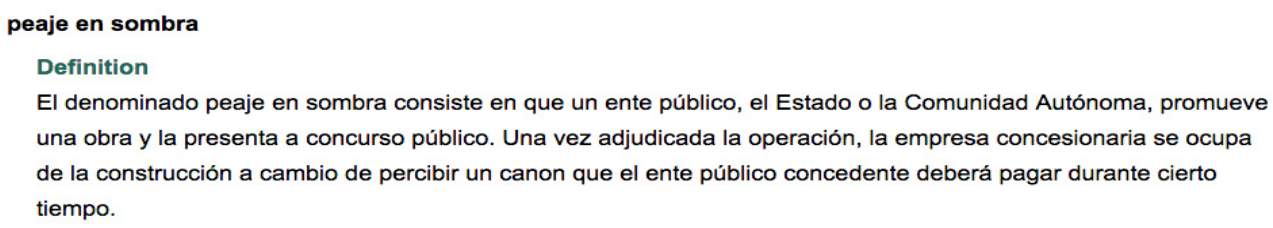

Figura 2: Peaje en la sombra en el Diccionario español de contabilidad

Los cambios en la terminología contable pueden ser también debidos a cambios culturales (en esta categoría se incluyen los cambios culturales con origen en el uso de idiomas diferentes). Cuando culturas distintas entran en contacto se ponen de manifiesto diferencias que han de ser estudiadas por los expertos para facilitar la transición y el acceso a los nuevos términos o conceptos a los profesionales y al público en general. Por ejemplo, la diferencia entre depreciación y amortización no existe en la contabilidad española (sí existe en la contabilidad inglesa). En la definición de la figura 3 observamos que el Diccionario español de contabilidad registra este hecho (no sería necesario para el experto español) porque puede ser útil para un traductor español-inglés de textos contables:

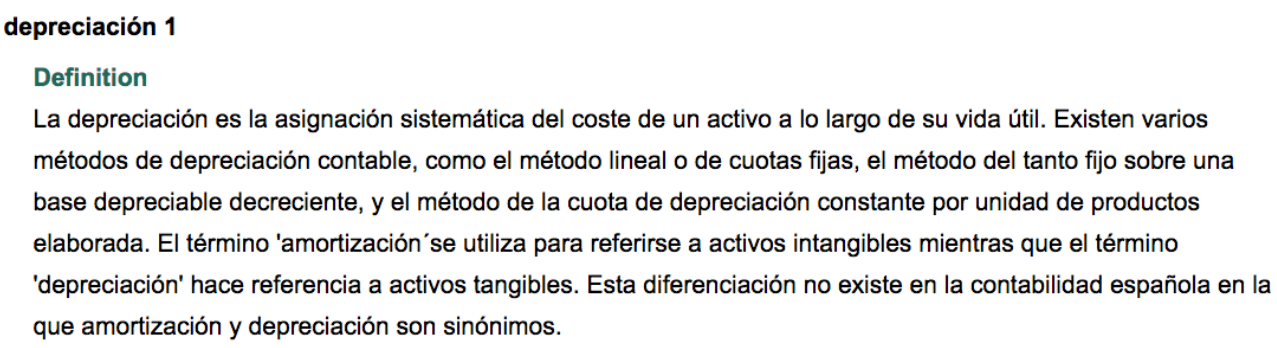

Figura 3: Depreciación en el Diccionario español de contabilidad

Aunque la necesidad de nuevos términos no tiene por qué, necesariamente, estar motivada por circunstancias totalmente novedosas; la evolución histórica de la sociedad puede originar percepciones distintas de la realidad económica y hacer aconsejable acuñar nuevos términos para describir mejor las evoluciones y cambios en el concepto (véase la figura 2). 
Los componentes técnicos de la contabilidad no pueden entenderse aisladamente del contexto sociocultural (incluyendo la influencia extranjera) en el que ocurren. Esto se debe a que la contabilidad es una construcción social, y aunque se pretenda una aplicación neutral, debe tenerse en cuenta el interés legítimo de personas y organizaciones en la elaboración de la información para la toma de decisiones.

El lenguaje puede ser una herramienta para la consecución de este fin informativo y para la mejor satisfacción de las necesidades informativas de los usuarios de la misma; pero debemos considerar que el relativismo lingüístico sugiere que el lenguaje que utilizamos puede influir en el pensamiento, o al menos en la percepción de la realidad económica que pretende describirse.

Un cambio en la terminología y/o en las definiciones de términos ya existentes puede pretender alterar las percepciones de la realidad observada puesto que puede ser utilizada para redefinir conceptos básicos. El crear o cambiar términos y conceptos como consecuencia de cambios culturales puede conllevar unas modificaciones más profundas que las ya mencionadas, porque las nuevas definiciones para términos nuevos pueden ser de tal calado que afecten a estructuras básicas del proceso contable.

Cuando los cambios en la terminología están motivados por la interrelación de idiomas distintos debe tenerse en cuenta que la adopción de los nuevos términos - a veces directamente como importación de estos idiomas y otras veces «traducidos» de esos mismos idiomas que además, generalmente, se imponen y superponen al idioma natural - propician la aparición de vocablos que aun con el mismo concepto pueden inducir a errores, sobre todo para los no expertos, porque el vocablo traducido o directamente importado no se corresponde con el significado que instintivamente le atribuimos en su uso común (véase la figura 3 ).

El uso de términos técnicos para comunicar la información contable puede conducir a malentendidos cuando el significado de esos términos no está totalmente apreciado por el destinatario de la información. La traductología sugiere que la plena equivalencia en la traducción entre lenguas es rara. Esto sugiere que el riesgo de malentendidos se agrava cuando los términos técnicos se traducen a otro idioma. Los errores de traducción de términos técnicos en el contexto de las teorías de la lingüística, que sugieren que el lenguaje influye en la manera en que pensamos. Se llega a la conclusión de que la elección de una etiqueta inapropiada en la traducción de la terminología contable es perjudicial para la comunicación internacional de contabilidad y crea problemas para los usuarios y los preparadores de los estados financieros traducidos, así como para los investigadores en contabilidad internacional y para los que participan en el proceso de armonización y normalización de la contabilidad (Labardin y Nikitin 2009).

Si el término es adoptado de una lengua extranjera (préstamo semántico), puede ocurrir que la terminología prestada y el significado no coincidan, es decir, no sean equivalentes de los originales. En este caso se produce una situación de conflicto que se resuelve con la implementación del término que corresponde al idioma dominante (Evans 2004: 211). Evidentemente, idioma dominante, en el momento actual, hace referencia al inglés por ser la lingua franca internacional, no solo en la contabilidad sino también en la ciencia y el mundo académico en general. En contabilidad la situación de privilegio 
del inglés se ve reforzada, además, por ser la lengua de las normas internacionales de contabilidad, con la situación de dominio que esto conlleva en la implantación obligada de términos y definiciones contables, enunciados en inglés originalmente.

\section{LOS DICCIONARIOS ESPECIALIZADOS}

La Teoría Funcional define la lexicografía como «la teoría y práctica de las herramientas de uso que se caracterizan por su capacidad para facilitar un acceso rápido y fácil al tipo específico de datos de los cuales un tipo específico de usuarios pueden extraer el tipo específico de información que les permita satisfacer el tipo específico de necesidades (lexicográficamente relevantes) que tengan en un tipo específico de situación social extra-lexicográfica» (Bergenholtz y Tarp 2003, Fuertes-Olivera y Tarp 2014; Tarp 2008).

Esta definición tiene múltiples implicaciones: en primer lugar, supone la desvinculación de la Lexicografía de la Lingüística Aplicada y de otras ciencias con las que comparte objetivos e intereses, como la Terminología. La Lexicografía pasa a ser una disciplina multidisciplinar en la que la elaboración de las herramientas lexicográficas (sean o no diccionarios) es llevada a cabo por profesionales de diversos campos del conocimiento. Por ejemplo, la elaboración y difusión del Diccionario español de contabilidad requiere de la colaboración de expertos diferentes que cubran con garantía suficiente las necesidades surgidas durante el proceso de construcción del diccionario. Lo que no significa que los criterios lingüísticos desaparezcan del plan de obra de los lexicógrafos sino que se incluirán siempre y cuando tengan un significado funcional, es decir, cuando sirvan al desempeño de las funciones que esta teoría asigna a la obra lexicográfica.

La lexicografía se presenta, pues, como una materia independiente que se ocupa del análisis y de la construcción de diccionarios con fines eminentemente prácticos, focalizando la atención no tanto en los usuarios reales del diccionario como en los usuarios potenciales considerando que para satisfacer las necesidades de los mismos es preciso el análisis pragmático de las situaciones social extralexicográficas en las que previsiblemente este usuario potencial pudiera encontrarse.

Un diccionario especializado está elaborado — si se atiende a los postulados básicos de la teoría funcional - para atender las necesidades de usuarios potenciales en distintas situaciones que puedan encontrarse. Las consideraciones principales a este respecto son dos: el perfil de usuario y las necesidades especiales del grupo de usuarios, ya que el interés principal del diccionario especializado es el de satisfacer necesidades lexicográficas diversas, a diferentes usuarios, de la forma más efectiva posible.

Las necesidades de los usuarios están inevitablemente ligadas al nivel de conocimiento de los lectores potenciales, que tienen un contexto situacional concreto y que participan en actividades diversas (estudiantes, traductores, revisores de textos, etc.); necesidades que pueden y deben ser facilitadas y cubiertas por los datos lexicográficos recogidos y organizados ya desde su inicio con el fin concreto de satisfacer estas necesidades. Los parámetros teóricos que tratan del perfil de usuario - situación y necesidades de los usuarios - deberán reflejarse en la información que se proporciona y en la 
forma de presentación de esta información, es decir, en la forma en que la misma está organizada y estructurada (Bergenholz y Nielsen 2006). Es necesario señalar que no todos los interesados en la disciplina tratada en el diccionario han de ser expertos en la materia, aunque sí mostrar un interés personal o profesional en la misma, el suficiente para generar una necesidad real de obtención o ampliación de estos conocimientos.

Desde esta perspectiva diccionarios como el Diccionario español de contabilidad suponen un avance notable sobre otros diccionarios especializados - por ejemplo, el Accounting Dictionary de Sánchez (2003) o diversos diccionarios de acceso libre en internet. Estos diccionarios continúan ofreciendo solamente o bien unos pocos datos lingüísticos (lema y equivalente en el ejemplo 1) o conceptuales (una definición con datos enciclopédicos en el ejemplo 2). En vez de ello, el Diccionario español de contabilidad incluye datos lingüísticos y conceptuales que incluyen definición, información gramatical, colocaciones y ejemplos de cada uno de los cuatro significados descritos en el diccionario (ejemplo 3):

amortization amortización

Ejemplo 1: La entrada amortization en el Accounting Dictionary (Sánchez 2003)

Amortización: Cancelación gradual de una deuda.

Ejemplo 2: La entrada amortización en el Diccionario de contabilidad de Rondón 
amortización nombre femenino

1 Definition

La amortización es la reducción gradual del coste de un activo intangible mediante la asignación sistemática de la cantidad amortizable del activo a lo largo de su vida útil.

Collocations

- amortización anual

- amortización semestral

- amortización de los activos financieros

- amortización de un préstamo

Examples

- La amortización de los activos intangibles es obligatoria.

2 Definition

La amortización es la devolución del principal de una deuda o de un título antes de o al vencimiento.

Collocations

- amortización de bonos

- la amortización de las acciones preferentes convertibles

- la amortización de las acciones ordinarias pendientes

- pérdidas y ganancias asociadas con las amortizaciones de pasivos financieros

- una acción preferente que proporciona el derecho de amortización en una fecha específica

- demandar la amortización

- el derecho a requerir amortización

- un derecho incondicional para rehusar la amortización

- la prohibición contra la amortización

- amortizaciones acumulativas

- coste a pagar por la amortización

Examples

- La transacción se reconoce como equivalente a cancelación o amortización.

3 Definition

La amortización es el reembolso de parte o del total de una cantidad previamente recibida.

Collocations

- amortización del capital

- el tratamiento de la amortización

- la amortización de primas

- periodo de amortización

- tener prevista una amortización máxima

Examples

- Las amortizaciones se reconocen cuando su cobro es prácticamente cierto.

4 Definition

La amortización es la recuperación de los fondos invertidos en una empresa.

Collocations

- amortización de la inversión

Examples

- Renault ha previsto la amaortización de los fondos destinados a la financiación del proyecto de coche eléctrico.

Ejemplo 3. La entrada amortización en el Diccionario español de contabilidad (Fuertes-

Olivera et alii 2013) 
Es en este marco teórico en el que se justifica la creación de una introducción sistemática en tanto en cuanto se presenta como un componente externo al listado «A-Z», pero unido o relacionado con el mismo, que permite ofrecer el conocimiento y la destreza necesarias para comprender el campo de la contabilidad y trabajar en el mismo con un cierto grado de garantía y seguridad. Desde esta perspectiva queda claro que la Introducción Sistemática es también un componente necesario de los diccionarios usados en situaciones cognitivas, es decir, es un componente lexicográfico que explica la similitud teórica y práctica entre diccionario y enciclopedia justificando de esta forma la idea defendida en este artículo: el diccionario especializado es una herramienta de información que puede diseñarse y construirse para satisfacer tanto las necesidades lingüísticas como conceptuales de su usuario potencial.

\section{LA INTRODUCCIÓN SISTEMÁTICA EN EL DICCIONARIO ESPAÑOL DE CONTABILIDAD}

Considerando que la fuente de información principal sobre la materia específica la constituye el núcleo del diccionario — los lemas y su definición-, la introducción sistemática sirve de complemento al diccionario en ese proceso de prestar información adecuada al usuario. Para ello es preciso concretar el tipo de información que debe contener, el tipo de usuario al que se dirige y el mecanismo de acceso a la misma ya que de forma general el usuario de la introducción sistemática no tiene que coincidir plenamente con el usuario del diccionario especializado. Por ejemplo, el experto contable acudirá al diccionario para solventar dudas en relación con su especialidad, pero no precisará consultar la introducción sistemática, más allá de la pura curiosidad que le pueda asistir, ya que los conocimientos en ella contenidos le son perfectamente comprensibles puesto que pertenecen a su acervo profesional.

El usuario del diccionario puede tener dificultades en el uso del diccionario especializado debidas a tres cuestiones, fundamentalmente: a) desconocimiento general de la materia, b) desconocimiento de aspectos específicos y más técnicos y c) diferencias culturales que pueden surgir como consecuencia de una evolución histórica de la materia considerada y/o del uso de varios idiomas (Cancino 2008). A todas estas dificultades, inherentes a las propias características e idiosincrasia de la materia tratada, se unen actualmente varias nuevas: las grandes posibilidades de información, almacenamiento de información y acceso a la misma que ofrece Internet. La acumulación de datos ha aumentado a un ritmo vertiginoso y pueden revelarse como un arma de doble filo. Por una parte, permiten disponer de mucha y variada información, pero esto puede llevar a un exceso y acumulación de datos que, sin criterio de organización y uso, puede convertirse en un serio impedimento para lograr los objetivos pretendidos.

La Introducción Sistemática es un texto integrado en un diccionario que se construye como herramienta capaz de proporcionar al usuario conocimiento sobre un área o materia específica, de una forma organizada y con el propósito de poder acceder a una información de calidad y de la forma más eficiente posible.

Si se asume que el usuario del Diccionario español de contabilidad no tiene que ser un experto en la materia pero tiene una necesidad real de conocimientos sobre la misma, la introducción sistemática se configura a modo de manual o guía al usuario no experto, a fin de facilitarle el uso, manejo y entendimiento del discurso especializado. 
Puede definirse de forma general, como: «el componente externo al listado del diccionario, que confeccionado a modo de manual, se convierte en una guía de uso del componente conceptual del diccionario; con la que el usuario no experto puede adquirir un conocimiento adecuado de un dominio» (Fuertes-Olivera y Tarp 2011:101). En resumen, la Introducción Sistemática (IS) trata de:

- proporcionar información sobre una materia específica (la contabilidad)

- a usuarios específicos (que tendrán unas características propias y, aunque su acceso a la IS guardará una estrecha relación con las razones que les asista para acceder a los Diccionarios de contabilidad, no tienen por qué coincidir con los usuarios del diccionario, ni con los usuarios de la información contable),

- que pueden encontrarse en alguna de las situaciones funcionales que se han descrito.

Así entendida, en el diseño de la introducción sistemática deberemos apoyarnos en los enunciados axiomáticos fundamentales de la Teoría Funcional de la Lexicografía, que resumimos en los dos siguientes:

(i) Cualquier obra lexicográfica debe servir para satisfacer necesidades de información, de cualquier disciplina y que puedan tener tipos específicos de usuarios en situaciones igualmente específicas.

(ii) Las necesidades de información de los usuarios se resuelven dando acceso a datos lexicográficos explícitamente preparados, de los que el usuario podrá extraer la información requerida que luego podrá emplear en solventar y resolver las situaciones que provocaron las necesidades de conocimientos.

\subsection{Estructura y diseño}

La «introducción sistemática» se configura como un manual o compendio, que se incorpora como anexo a un diccionario especializado. En ella se lleva a cabo el estudio sistemático, detallado y organizado del campo concreto del que trata el diccionario, con el fin de reunir y destacar aquellos conceptos que se estimen básicos y elaborar estos conocimientos a partir de datos concretos o reales. Cumple, por tanto, una misión como herramienta formativa, que va más allá de la función comunicativa, ya que es capaz de proporcionar al usuario no experto conocimiento contable de forma clara, precisa y organizada. Se estructura a partir de conceptos básicos para ir construyendo sobre ellos y a partir de ellos, un entramado de conocimientos especializados, con la amplitud y desarrollo que se estime oportuno para el cumplimiento de los fines concretos que se le asignen.

Respecto al contenido, la introducción describe conceptualmente un campo de conocimiento específico determinado y está dirigida a unos usuarios con distintos grados de conocimiento de esa materia, por lo que deberá llevarse a cabo una recopilación, descripción y presentación de términos que sean elementos léxicos utilizados por los expertos y creados en ese ámbito específico.

Se trata para incluir en la introducción sistemática de identificar términos que definan la esencia de la contabilidad, elementos que sean definitorios de la esencia contable, considerando el estudio que de las características y objetivos se ha hecho de la 
contabilidad. Estos elementos son aquellos que han permanecido como tales en el tiempo, aún considerando como inevitable que esos términos básicos y esenciales no permanecen inmutables sino que evolucionan y se modifican en el tiempo y que pueden no ser términos concretos y creados expresamente para el campo específico estudiado, sino que pueden ser elementos existentes en otros ámbitos o materias afines.

Estos términos pueden estar constituidos por una o varias palabras que identifican de forma clara y precisa un concepto original que con independencia de las palabras utilizadas para definirlo y de las modificaciones o variaciones que puedan haberse producido, sea fácilmente reconocible por el experto y asequible al lego que deberá realizar una tarea de comprensión, más o menos intensa en función de los conocimientos previos, de las necesidades de información que tenga y de la motivación que le asista para lograr esa comprensión.

\subsection{Organización de la «Introducción Sistemática»}

En los diccionarios impresos, la introducción sistemática se ubicaría al inicio del diccionario junto con las instrucciones de uso y tabla de abreviaturas, signos y símbolos empleados en el diccionario, si fuera necesario. El contenido y estructura serían los mismos, en este formato, aunque por motivos obvios de espacio y coste debería procurarse limitar al mínimo comprensible las definiciones y explicaciones de la materia tratada. Aunque considerando la rapidez con la que los diccionarios en soporte digital están desplazando a los impresos, la referencia a estos tiene un mero carácter testimonial.

La información recogida en la IS está diseñada para su lectura continuada pero esta posibilidad no sería operativa por el tiempo que requeriría y porque puede no ser precisa esa lectura continua para el fin cognitivo pretendido. Debe tenerse en cuenta que «los productos lexicográficos son por definición herramientas de consulta puntual que solo se leen o estudian parcialmente en función de las necesidades puntuales y específicas que dan lugar a cada consulta» (Fuertes-Olivera y Tarp 2008: 77).

En un aspecto meramente formal, conviene recordar que la introducción sistemática se plantea como un documento conectado al Diccionario español de Contabilidad, por lo que debe atender a aspectos metodológicos contemplados para los mismos, en concreto lo establecido por la Teoría Funcional de la Lexicografía. Así, en su formulación se tendrán en cuenta los aspectos siguientes:

El contenido se estructura de forma tal que pueda accederse a él para ser leído de forma continuada o fragmentada, en partes que deben tener un contenido conceptual independiente. Para ello se numerarán correlativamente los conceptos principales y sucesivamente se descenderá en esa numeración para ir ampliando contenido, pero permitiendo, a la vez, la interrupción de la lectura en el punto subordinado que se considere conveniente.

— En la redacción de las definiciones, se atenderá a su carácter divulgativo, evitando en lo posible los tecnicismos y también las referencias cruzadas, es decir, debe evitarse al usuario consultas adicionales para su entendimiento. Esta consulta a otras fuentes, sin embargo, sí está prevista pueda realizarse en la consulta del listado A-Z de los diccionarios. 
- En la introducción sistemática no se hace referencias a cuestiones lingüísticas del término o concepto definido. Estas cuestiones sí tienen cabida para el mismo término cuando, en el uso del diccionario se acceda a él como entrada independiente.

- Se incluyen aquellos términos cuyo concepto sea sobradamente conocido por el experto contable. Es decir, aquellos términos esenciales, cuya permanencia en el tiempo haya supuesto que sean asumidos y reconocidos intuitivamente por el experto, ya que son básicos e imprescindibles en el conocimiento de la contabilidad. Este conocimiento permite al contable reconocer conceptos aunque la denominación de los mismos haya cambiado, consecuencia de evoluciones culturales y/o imposiciones idiomáticas $\mathrm{y}$, por el contrario, le permite reconocer que el concepto es distinto aunque haya permanecido la nominación del término.

- La redacción se realiza utilizando expresiones específicas y concretas; tendiendo, en la medida que sea posible, a que el contenido forme parte del conocimiento natural del experto contable; sin que el mismo pueda identificarse con una situación, norma y emisor concreto. Es decir, nadie puede pretender la autoría de lo expresado, aunque al profesional contable le sea perfectamente reconocible la materia expuesta.

- El contenido debe cumplir con dos requisitos: debe ser sobradamente conocido para el experto contable y debe ser entendido, con un mínimo esfuerzo comprensivo, por el no contable. Todo ello sin perder el rigor técnico y conceptual que tiene la contabilidad.

- Los términos y conceptos recogidos son universales; el lenguaje técnico contable está aceptado como tal en cualquier idioma y época. Por lo que, con independencia del idioma en el que se redacte originalmente, son fácilmente trasladables a otros idiomas. Este hecho hace posible que la introducción sistemática propuesta pueda ser añadida a los diccionarios de contabilidad redactados en diferentes idiomas, porque por corresponderse con conceptos y términos fácilmente reconocibles por el experto, no deben suponer ninguna dificultad en su traducción.

- La numeración realizada de los términos principales con un solo digito y de las divisiones o sub-epígrafes con dos dígitos responde al hecho de que, esta clasificación permite una lectura continuada con el acceso a los epígrafes y subepígrafes numerados correlativamente o interrumpiendo su lectura, y reanudando la misma en el mismo epígrafe o en otro posterior, según estime conveniente.

\section{CONCLUSIONES}

En estos tiempos en los que la progresión del conocimiento avanza de un modo imparable y en los que se dispone de soportes diversos para su difusión, ni siquiera los diccionarios cognitivos, por más exhaustivos que puedan ser en cuanto a elaboración y contenidos, no pueden abarcarlo todo; pero sí que pueden sintetizar, organizar y presentar la información más que voluminosa que puedan recoger, buscando siempre en primer plano y atendiendo a lo esencial y perdurable de la materia. Los diccionarios especializados deben atender a las necesidades cognitivas de sus usuarios, es decir, 
deben permitir a los usuarios obtener o aumentar sus conocimientos en la materia principal sobre la que versa ese diccionario.

Los diccionarios atienden y resuelven problemas de usuarios diferentes con necesidades diferentes. El experto necesitará acudir al diccionario para consultar términos y expresiones que por novedosas, o por encontrarse en idiomas distintos en diccionarios bilingües, pueda no conocer. El lego o el semiexperto también precisará del diccionario para resolver problemas diversos, pero además de forma expresa o por remisión desde una entrada tendrá acceso a conocimiento contable en la introducción sistemática más básico, más accesible a sus conocimientos.

La introducción sistemática resuelve dificultades de uso del diccionario originadas por la falta de conocimiento del usuario, ya que su contenido versa sobre aspectos esenciales y definitorios del conocimiento contable que por permanecer en el tiempo superan los conflictos culturales que se presentan por diferencias de idiomas o falta de conocimiento conceptual básico de la materia que es objeto del diccionario especializado.

\section{REFERENCIAS BIBLIOGRÁFICAS}

AlLAN, K. $\left(2006^{2}\right)$ : «Jargon», en K. Brown, ed., Encyclopedia of Language \& Linguistics, Amsterdam-Boston, Elsevier, vol. 6, pp. 109-112.

ARrington C. E y J. E. FrANCIS (1993): «Giving economic accounts: accounting as cultural practice», Accounting, Organizations and Society, 18, 2-3, pp. 107-124.

AVERY, H. (1953): «Accounting as a language», The Accounting Review, 28, 1, pp. 83-87.

BelKaOUI, A. (1978): «Linguistic relativity in accounting», Accounting, Organizations and Society, 3, 2, pp. 97-104.

BelKaOUI, R. A. (1980): «The interprofessional linguistic Communications of accounting Concepts: An experiment in sociolinguistic», Journal of Accounting Research, 18, 2, pp 362-374.

BERGENHOLTZ H. y S. TARP (2003): «Two opposing theories: On H.E. Wiegand's recent discovery of lexicographic functions», Hermes: Journal of Linguistics, 31, pp. 171-196.

Besomi, D. (2011): Crises and Cycles in Economic Dictionaries and Encyclopedias, London-New York, Routledge.

BlanK, A. (1999): «Why Do New Meanings Occur? A Cognitive Typology of the Motivations for Lexical Semantic Change», en A. Blank y P. Koch, eds., Historical Semantics and Cognition, Berlin-New York, Mouton de Gruyter, pp. 61-89.

Bотнма, T. (2015): «Reflexiones sobre la interrelación entre lexicografía y ciencia de la información: una perspectiva personal», Estudios de Lexicografia, 4, pp. 21-30.

Burchell, S., C. ClubB, A. Hopwood, J. Hughes y J. Nahapiet (1980): «The roles of accounting in organizations and society», Accounting, Organizations and Society, 5, 1, pp. 5-27.

CANCINO, R. (2008): «Mejor ser heredero en Dinamarca que en España: la integración de las diferentes culturas en diccionarios bilingües. Una propuesta para la introducción de información enciclopédica en el diccionario jurídico bilingüe», en D. Azo- 
rín, ed., El diccionario como puente entre las lenguas y culturas del mundo. Actas del II Congreso Internacional de Lexicografía Hispánica, Alicante, Biblioteca Virtual Miguel de Cervantes, pp. 622-627.

Cuevas MejíA, J. J (2010): «La contabilidad como lenguaje: Una mirada institucional a su contribución en la formación del sujeto- organización», Contaduría Universitaria de Antioquia, 57, pp. 37-50.

EngBerg, J. $\left(2006^{2}\right)$ : «Languages for Specific Purposes», en K. Brown, ed., Encyclopedia of Language \& Linguistics, Amsterdam, Boston, Elsevier, vol. 6, pp. 679-684.

EvANS, L. (2004): «Language, translation and the problem of International accounting Communications», Accounting, Auditing \& Accountability Journal, 17, 2, pp 210-248.

Fuertes-OliverA, P. A. y M. NiÑO AMO (2011): «Internet dictionaries for communicative and cognitive functions: El diccionario inglés-español de contabilidad», en P. A. Fuertes-Olivera y H. Bergenholtz, eds, e-Lexicography, the Internet and Digital Initiatives, London, Continuum, pp.168-186.

Fuertes Olivera, P. A. y S. TARP (2008): «La Teoría funcional de la Lexicografía y sus consecuencias para los diccionarios de economía del español», Revista de Lexicografía, XIV, pp.75-95.

Fuertes-Olivera, P. A. y S. TARP. (2011): «Lexicography for the Third Millennium: Cognitive-oriented Specialised Dictionaries for Learners», Ibérica: Journal of the European Association of Languages for Specific Purposes, 21, pp.141-162.

Fuertes-Olivera, P. A. y S. TARP (2014): Theory and Practice of Specialised Online Dictionaries. Lexicography versus Terminography, Berlin-New York, De Gruyter.

GÓMEZ DE ENTERRÍA, J. (2000): «Los diccionarios especializados y la enseñanza de ELE», en M. A. Martín Zorraquino, ed., ¿Qué español enseñar?: norma y variación lingüisticas en la enseñanza del español a extranjeros. Actas XI Congreso de Asociación para la enseñanza del español como lengua extranjera, Zaragoza, Universidad de Zaragoza-ASELE, pp. 105-122.

Gutiérrez Rodilla, B. (1998): La ciencia empieza en la palabra. Análisis e historia del lenguaje científico, Barcelona, Península.

HARIED, A. A (1972): «The semantics dimensions of Financial Reports», Journal of Accounting Research, 10, 2, pp. 376-391.

IRAUSQUín, C (2012): «Perspectivas hermenéuticas como metodología en la investigación de las ciencias contables», Multiciencias, 12, 2, pp. 167-173.

JAyAsinghe, K., T. SoOBAROYEn y D. Thoma (2001): «Accounting as the "language" of organisational», International Journal of Critical Accounting, 3, 1, pp. $26-62$.

KILliAN, S. (2010): «No accounting for these people: Shell in Ireland and accounting language», Critical perspectivas on Accounting, 21, 8, pp. 711-723.

LABARDin, P. y M. NiKitin (2009): «Accounting and the Words to Tell It: an Historical Perspective», Accounting, Business \& Financial History, 19, 2, pp. 149-166.

LeÓn Araúz, P., P. FABer y C. PÉREZ HeRnÁNDEZ (2008): «LSP dictionaries and their genuine purpose: a frame-based example from MARCOCOSTA», en J. DeCesaris y E. Bernal, eds., Proceedings of the XIII EURALEX International Congress (Barcelona, 15-19 July 2008), Barcelona, Universitat Pompeu Fabra, pp.997-1006

LÓPEZ PÉREZ, M. ${ }^{a}$ V. (1999): «Captación e interpretación en Contabilidad», Revista Española de Financiación y Contabilidad, 28, 102, pp. 1181-1184 
LÓPEZ PÉREZ, M. ${ }^{a}$ V. y L. RodríGuez ArIZA (2002): «Aplicación del enfoque cognitivo a la metodología contable», Revista Española de Financiación y Contabilidad, 31, 112, pp. 461-493.

Oliver, B. L. (1974): «The Semantic Differential: a Device for Measuring the Interprofessional Communication of Selected Accounting Concepts», Journal of Accounting Research, 12, 2, pp. 299-316.

PARKER, R. H. (1994): «Finding English Words to Talk about Accounting Concepts», Accounting, Auditing \& Accountability Journal, 7, 2, pp. 70-85.

PINGER, R. W (1954): «The semantics of accounting», The Accounting Review, 29, 4, pp. 652-655.

Preston, A. (1986): «Interactions and arrangements in the process of informing», Accounting, Organizations and Society, 11, 6, pp. 521-540.

Rueda Delgado, G. (2011): «Democracia, emancipación y contabilidad», Papel Político, 16,1, pp. 122-135.

TARP, S. (2008): Lexicography in the Borderland Between Knowledge and Non-knowledge, Tübingen, Niemeyer.

TARP, S. (2015): «La teoría funcional en pocas palabras», Estudios de Lexicografía, 4, pp. 31-42.

TuA PeredA, J. (2004): «El marco conceptual, soporte de las Normas Internacionales», AECA: Revista de la asociación de Contabilidad y Administración de Empresas, 66, pp. 2-8.

Wilkinson, T. L. (1964): «Can Accounting Be An International Language?», The Accounting Review, 39, 1, pp.133-139.

WhORF, B. L. (1956): Language, Thought, and Reality, Cambridge, Massachusetts, MIT Press.

\section{DICCIONARIOS}

Fuertes-Olivera, P. A., H. Bergenholtz, S. Nielsen, M. Niño Amo, P. Gordo Gómez, Á. De los Ríos Rodicio, Á. Sastre Ruano y M. Velasco Sacristán (2013): Diccionario español de contabilidad, Hamburgo, Lemma.com.

RONDÓN, J. Diccionario de Contabilidad. (http://diccionariodecontabilidadd.blogspot.com.es/)

SÁnchez, N. (2003): Accounting dictionary. Diccionario de contabilidad inglés-español, español-inglés, español-español, Hoboken, John Wiley and Sons.

TÍTULO/TitLe

«La Introducción Sistemática en el Diccionario Especializado» «Systematic Introductions in Specialised Dictionaries» 
Resumen: Este artículo tiene dos objetivos fundamentales. En primer lugar, se lleva a cabo una reflexión teórica sobre el diccionario especializado y su construcción. Dicha reflexión sitúa el artículo en el marco teórico de la Teoría Funcional de la Lexicografía que, entre otras cosas, defiende que los diccionarios especializados son herramientas construidas para solucionar problemas puntuales de información en diferentes situaciones de uso, por ejemplo, situaciones comunicativas (traducción) o cognitivas (estudio y reflexión). En segundo lugar, se ponen en práctica algunas de la reflexiones teóricas anteriores explicando los pasos llevados a cabo para construir una introducción sistemática que va a ser incluida en el Diccionario español de contabilidad como componente lexicográfico necesario para explicar las características conceptuales de un campo del saber a semiexpertos y legos y para ilustrar algunos de los usos lingüísticos de los términos del campo. Por razones de espacio nos centraremos en el componente conceptual o enciclopédico del diccionario lo que, entre otras cosas, nos obliga a precisar los fundamentos de la contabilidad, su caracterización como lenguaje de especialidad y la relación de la introducción sistemática con el listado principal del diccionario.

Palabras clave: Teoría Funcional de la Lexicografía; introducción sistemática; español; diccionario; contabilidad.

Abstract: The main objectives of this paper is, firstly, to discuss the theoretical foundations on which specialised dictionaries can be built, and secondly, to put some of them into practice, e.g. commenting on decisions taken in relation with the construction of a systematic introduction to be included in the Diccionario español de contabilidad. Regarding the first objective, this article is based on the tenets of the Function Theory of Lexicography, which, among other issues, defends that specialised dictionaries are tools for satisfying punctual information needs in several use situations. For instance, they can contain both linguistic and conceptual data thus eliminating the traditional distinction between dictionary and encyclopaedia. This can be done by incorporating a systematic introduction, i.e. a dictionary component that aims at providing help in cognitive-oriented and communicativeoriented user situations. In the former type of situations, the component provides an introduction and detailed presentation of a subject field. In the communicative-related situations, the systematic introduction supplements the encyclopaedic information with language information in the form of collocations, auxiliary words and examples of usage. For reasons of space, we will focus on the cognitive component of a systematic introduction, which, among other things, will force us to explain the foundations of accounting, its role as specialised language, and its relaytionship with the A-Z list of the dictionary.

Key words: Function Theory of Lexicography; systematic introduction; Spanish; dictionary, accounting. 